\title{
Domination Number of Square of Cartesian Products of Cycles
}

\author{
Morteza Alishahi, Sakineh Hoseini Shalmaee \\ Islamic Azad University, Nazarabad Branch \\ Email: morteza.alishahi@gmail.com
}

Received 2 August 2015; accepted 26 October 2015; published 29 October 2015

Copyright (C 2015 by authors and Scientific Research Publishing Inc.

This work is licensed under the Creative Commons Attribution International License (CC BY). http://creativecommons.org/licenses/by/4.0/

(c) () Open Access

\begin{abstract}
A set $S \subseteq V(G)$ is a dominating set of $G$ if every vertex of $V(G)-S$ is adjacent to at least one vertex of $S$. The cardinality of the smallest dominating set of $G$ is called the domination number of $G$. The square $G^{2}$ of a graph $G$ is obtained from $G$ by adding new edges between every two vertices having distance 2 in $G$. In this paper we study the domination number of square of graphs, find a bound for domination number of square of Cartesian product of cycles, and find the exact value for some of them.
\end{abstract}

Keywords

Domination Number, Square of a Graph, Cartesian Product

\section{Introduction}

The usual graph theory notions not herein, refer to [1]. The neighborhood of vertex $u$ is denoted by $N(u)=\{v \in V(G): u v \in E(G)\}$ and the close neighborhood of vertex $u$ is denoted by $N[u]=N(u) \cup\{u\}$. Let $S \subseteq V(G)$, the neighborhood and closed neighborhood of $S$ are defined as $N(S)=\bigcup_{u \in S} N(u)$ and $N[S]=\bigcup_{u \in S} N[u]$. If $u \in V(G)$, then $N_{k}(u)=\{v \in V(G) \mid 1 \leq d(u, v) \leq k\}$. If $S \subseteq V(G)$ and $u \in V(G)$, then $d(u, S)=\min \{d(u, v) \mid v \in S\}$. The diameter of $G$ denoted by $\operatorname{diam}(G)$ is defined as $\operatorname{diam}(G)=\max _{u, v \in V(G)} d_{G}(u, v)$. A set $S \subseteq V(G)$ is a dominating set of $G$ if every vertex of $V(G)-S$ is adjacent to at least one vertex of $S$. The cardinality of the smallest dominating set of $G$, denoted by $\gamma(G)$, is called the domination number of $G$. A dominating set of cardinality $\gamma(G)$ is called a $\gamma$-set of $G$ [2]. A dominating set $S$ is a minimal dominating set if no proper subset $S^{\prime} \subset S$ is a dominating set. Given any graph $G$, its square graph $G^{2}$ is a graph with vertex set $V(G)$ and two vertices are adjacent whenever they are at 
distance 1 or 2 in $G$. For example $C_{5}^{2}=K_{5}$. A set $S \subseteq V(G)$ is a 2-distance dominating set of $G$ if $d_{G}(u, S)=1$ or 2 for every vertex of $V(G)-S$. The cardinality of the smallest 2-distance dominating set of $G$, denoted by $\gamma^{2}(G)$, is called 2-distance domination number of $G$. Every 2-distance dominating set of $G$ is a dominating set of $G^{2}$, so $\gamma^{2}(G)=\gamma\left(G^{2}\right)$. The Cartesian product of Graphs $G$ and $H$ denoted by $G \square H$ is a graph with vertex set $V(G \square H)=V(G) \times V(H)$ and the edge set

$E(G \square H)=\{((u, v),(z, w)):(u z \in E(G) \& v=w)$ or $(u=z \& v w \in E(H))\}$. The graph $G \square H$ is obtained by locating copies $H_{i}$ of grpah $H$ instead of vertices of $G$ and connecting the corresponding vertices of $H_{i}$ to $H_{j}$ if vertex $v_{i}$ is adjacent to $v_{j}$ in $G . G \square H$ is isomorphic to $H \square G$. We denote a cycle with $n$ vertices by $C_{n}$ and a path with $n$ vertices by $P_{n}$. The bipartite geraph $K_{1,3}$ is named claw.

\section{Preliminaries Results}

Theorem 1. Let $G$ be a graph. Then

a) If $u v \in E(G)$, then $\gamma(G-u v) \geq \gamma(G)$.

b) If $u v \in E\left(G^{c}\right)$, then $\gamma(G+u v) \leq \gamma(G)$.

Proof. a) Every dominating set of $G-u v$ is a dominating set of $G$ so $\quad \gamma(G-u v) \geq \gamma(G)$.

b) Every dominating set of $G$ is a dominating set of $G+u v$ so $\gamma(G+u v) \leq \gamma(G)$.

Theorem 2. [3] A dominating set $S$ is a minimal dominating set if and only if for each vertex $u \in S$, one of the following conditions holds:

a) $u$ is an isolated vertex of $S$.

b) there exist a vertex $v \in V(G)-S$ for which $N(v) \cap S=\{u\}$.

Theorem 3. [3] If $G$ is a graph with no isolated vertices and $S$ is a minimal dominating set of $G$, then $V(G)-S$ is a dominating set of $G$.

Proof. Let $S$ be a $\gamma$-set of $G$. $S$ is a minimal dominating set of $G$. By Theorem 3, $V(G)-S$ is a dominating set of $G$ too, so $|S| \leq|V(G)-S|$, so $|S| \leq \frac{n}{2}$.

Theorem 4. [4] If $G$ is a connected claw free graph, then $\gamma(G) \leq\left\lceil\frac{n}{3}\right\rceil$.

Theorem 5. [5] Let $G$ be a graph. Then $\left\lceil\frac{n}{1+\Delta(G)}\right\rceil \leq \gamma(G) \leq n-\Delta(G)$.

Since $\Delta\left(C_{n}\right)=\Delta\left(P_{n}\right)=2$, by Theorems 4 and 5 we have the following corollary.

Corollary 6. $\gamma\left(C_{n}\right)=\gamma\left(P_{n}\right)=\left\lceil\frac{n}{3}\right\rceil$.

Vizing conjecture

Let $G$ and $H$ be two graphs. Then $\gamma(G \square H) \geq \gamma(G) \gamma(H)$ [6].

\section{Domination Number of Square of Graphs}

Theorem 7. Let $S$ be a dominating set of $G^{2}$. Then $S$ is a minimal dominating set of $G^{2}$ if and only if each vertex $u \in S$ satisfies at least one of the following conditions:

a) There exists a vertex $v \in V(G)-S$ for which $N_{2}(v) \cap S=\{u\}$.

b) $d(u, w)>2$ for every vertex $w \in S-\{u\}$.

Proof. If $u \in S$ and $u$ does't satisfy conditions a) and b), then the set $S-\{u\}$ is a dominating set of $G^{2}$ that is contradiction. Conversely, let $S$ be a dominating set of $G^{2}$ but not minimal. Then there exists a vertex $u \in S$ such that $S-\{u\}$ is a dominating set of $G^{2}$, too. So $d(v, S-\{u\})=1$ or 2 for every $v \in V(G)-S$; therefore $S$ doesn't satisfy in condition a). In addition $d(u, S-\{u\})=1$ or 2 , so $S$ doesn't satisfy in condition b). 
Theorem 8. If $\operatorname{diam}(G) \leq 3$, then $\gamma\left(G^{2}\right) \leq \delta(G)$.

Proof. Let $d(u)=\delta(G)$. Since $\operatorname{diam}(G) \leq 3$, the set $N(u)$ is a dominating set of $G^{2}$. Therefore $\gamma\left(G^{2}\right) \leq|N(u)|=d(u)=\delta(G)$.

Theorem 9. If $\operatorname{diam}(G) \leq 4$, then $\gamma\left(G^{2}\right) \leq\left(\sum_{v \in N(u)} d(v)\right)-d(u)$, for every $u \in V(G)$.

Proof. Let $u$ be an arbitrary vertex of $G$. Let $S(u)=N(N(u))-\{u\}$. Since $\operatorname{diam}(G) \leq 4, d(v, S(u)) \leq 2$, for every $v \in V(G)$. Therefore $S(u)$ is a dominating set of $G^{2} .|S(u)| \leq \sum_{v \in N(u)}(d(v)-1)$ and $|N(u)|=d(u)$, Hence $\gamma\left(G^{2}\right) \leq|S(u)| \leq \sum_{v \in N(u)}(d(v)-1)=\sum_{v \in N(u)}(d(v))-d(u)$.

Theorem 10. Let $G$ be a graph. Then $\gamma\left(\left(G \square K_{n}\right)^{2}\right)=\gamma(G)$.

Proof. Let $V(G)=\left\{u_{1}, u_{2}, \cdots, u_{m}\right\}$ and $H_{1}, H_{2}, \cdots, H_{m}$ be the copies of $K_{n}$ in $G \square K_{n}$ corresponding to the vertices $u_{1}, u_{2}, \cdots, u_{m}$. Let $S=\left\{u_{t 1}, u_{t 2}, \cdots, u_{t k}\right\}$ be a $\gamma$-set of $G$. Then the set $S^{\prime} \subseteq V\left((G \square H)^{2}\right)$ that contains a vertex of each copies $K_{t 1}, K_{t 2}, \cdots, K_{t k}$ is a $\gamma$-set of $\left(G \square K_{n}\right)^{2}$. Since $\left|S^{\prime}\right|=|S|$, the result holds.

Theorem 11. For every $n \geq 3, \gamma\left(C_{n}^{2}\right)=\left\lceil\frac{n}{5}\right\rceil$.

Proof. The graphs $C_{3}^{2}$ and $C_{4}^{2}$ are complete graphs, therefore $\gamma\left(C_{3}^{2}\right)=\gamma\left(C_{4}^{2}\right)=1$. So the result holds for $C_{3}^{2}$ and $C_{4}^{2}$. Let $C_{n}=u_{1} u_{2} \cdots u_{n} u_{1}, n \geq 5$. Since $\Delta\left(C_{n}^{2}\right)=4$, by the Theorem 5 we have $\gamma\left(C_{n}^{2}\right) \geq\left\lceil\frac{n}{5}\right\rceil$. On the other hand by Figure 1 the set $S=\left\{u_{5 k+1}: k=0,1, \cdots,\left\lceil\frac{n}{5}\right\rceil-1\right\}$ is a dominating set of size $\left\lceil\frac{n}{5}\right\rceil$ for $C_{n}^{2}$. So $\gamma\left(C_{n}^{2}\right) \leq\left\lceil\frac{n}{5}\right\rceil$, therefore $\gamma\left(C_{n}^{2}\right)=\left\lceil\frac{n}{5}\right\rceil$.

Theorem 12. For every $n \geq 1, \gamma\left(P_{n}^{2}\right)=\left\lceil\frac{n}{5}\right\rceil$.

Proof. $\gamma\left(P_{1}^{2}\right)=\gamma\left(P_{2}^{2}\right)=\gamma\left(P_{3}^{2}\right)=\gamma\left(P_{4}^{2}\right)=1$, and the result holds for these graphs. Let $P_{n}=u_{1} u_{2} \cdots u_{n}$, $n \geq 5$. Since $\Delta\left(P_{n}^{2}\right)=4$, by Theorem 5 we have $\gamma\left(P_{n}^{2}\right) \geq\left\lceil\frac{n}{5}\right\rceil$. By Figure 2 the set:

$$
S= \begin{cases}\left\{u_{5 k+3}: k=0,1, \cdots,\left\lfloor\frac{n}{5}\right\rfloor-1\right\} & \text { if } n \equiv 0(\bmod 5) \\ \left\{u_{5 k+3}: k=0,1, \cdots,\left\lfloor\frac{n}{5}\right\rfloor-1\right\} \bigcup\left\{u_{n}\right\} & \text { if } n \equiv 1,2,3(\bmod 5) \\ \left\{u_{5 k+3}: k=0,1, \cdots,\left\lfloor\frac{n}{5}\right\rfloor-1\right\} \bigcup\left\{u_{n-1}\right\} & \text { if } n \equiv 4(\bmod 5)\end{cases}
$$

is a dominating set of size $\left\lceil\frac{n}{5}\right\rceil$ for $P_{n}^{2}$, so $\gamma\left(P_{n}^{2}\right) \leq\left\lceil\frac{n}{5}\right\rceil ;$ therefore $\gamma\left(P_{n}^{2}\right)=\left\lceil\frac{n}{5}\right\rceil$.

Theorem 13. For every $m, n \geq 1, \gamma\left(\left(P_{m} \square P_{n}\right)^{2}\right) \geq\left\lceil\frac{m n}{13}\right\rceil$, and for every $m, n \geq 3, \gamma\left(\left(C_{m} \square C_{n}\right)^{2}\right) \geq\left\lceil\frac{m n}{13}\right\rceil$.

Proof. The graphs $P_{m} \square P_{n}$ and $C_{m} \square C_{n}$ have $m n$ vertices and every vertex $u$ dominates at least 13 vertices in $\left(P_{m} \square P_{n}\right)^{2}$ and $\left(C_{m} \square C_{n}\right)^{2}$ (Figure 3), so the result holds.

By Theorem 13, $\gamma\left(\left(P_{m} \square P_{n}\right)^{2}\right)$ or $\gamma\left(\left(C_{m} \square C_{n}\right)^{2}\right)$ equals the minimum number of diamonds like Figure 4. we can cover all the vertices of $P_{m} \square P_{n}$ or $C_{m} \square C_{n}$. 


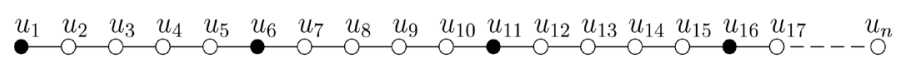

Figure 1. A dominating set of $C_{n}^{2}$.

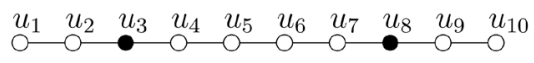

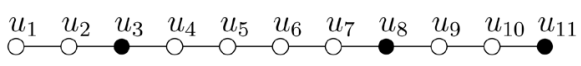

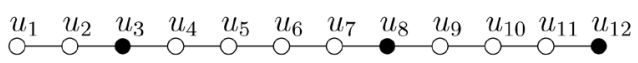

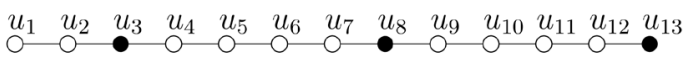

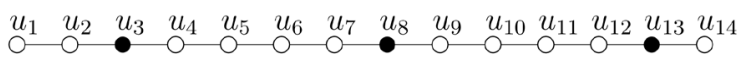

Figure 2. A dominating set of $P_{n}^{2}$.

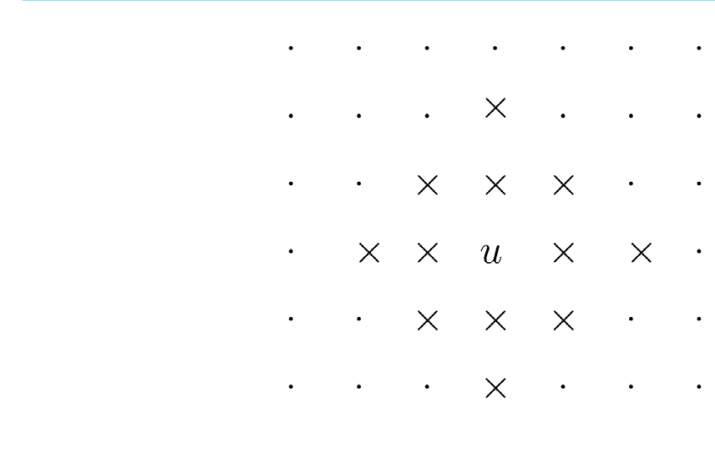

Figure 3. Dominated vertices by $u$ in $\left(P_{m} \square P_{n}\right)^{2}$ and $\left(C_{m} \square C_{n}\right)^{2}$.<smiles>[Li][13CH3]</smiles>

$\times \quad \times \quad x$

$x \times x \times x$

$\times \quad \times \quad x$

X

Figure 4. Dominated vertices by one vertex in $\left(P_{m} \square P_{n}\right)^{2}$ and $\left(C_{m} \square C_{n}\right)^{2}$.

In this paper we use short display or $s . d$ to show the graphs $P_{m} \square P_{n}$ and $C_{m} \square C_{n}$ for simplicity; it means that we don't draw the edges of these graphs and draw only their vertices.

Theorem 14. For every $k, t \geq 1, \gamma\left(\left(C_{13 k} \square C_{13 t}\right)^{2}\right)=13 k t$.

Proof. By Theorem 13 we have $\gamma\left(\left(C_{13} \square C_{13}\right)^{2}\right) \geq 13$. In Figure 5 that is $s . d$ of $C_{13} \square C_{13}$. It is determined by a $\gamma$-set of size 13 for $\left(C_{13} \square C_{13}\right)^{2}$. Therefore $\gamma\left(\left(C_{13} \square C_{13}\right)^{2}\right) \leq 13$; hence $\gamma\left(\left(C_{13} \square C_{13}\right)^{2}\right)=13$.

We can obtain s.d of $C_{13 k} \square C_{13 t}$ with dominating set of size $13 k t$ for $\left(C_{13 k} \square C_{13 t}\right)^{2}$ by locating $k t$ copies of Figure 5 in $k$ rows and $t$ columns. Hence $\gamma\left(\left(C_{13 k} \square C_{13 t}\right)^{2}\right) \leq 13 k t$. By Theorem 13 we have 


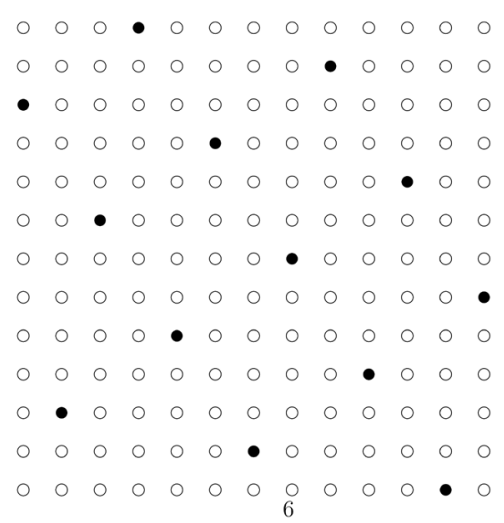

Figure 5. A dominating set of size 13 for $\left(C_{13} \square C_{13}\right)^{2}$.

$\gamma\left(\left(C_{13 k} \square C_{13 t}\right)^{2}\right) \geq 13 k t$, so $\gamma\left(\left(C_{13 k} \square C_{13 t}\right)^{2}\right)=13 k t$

Theorem 15. $\gamma\left(\left(C_{3} \square C_{n}\right)^{2}\right)=\left\lceil\frac{n}{3}\right\rceil$, for every $n \geq 3$.

Proof. Since $C_{3}=K_{3}$, by Theorem 10 and Corollary 6 we have

$$
\gamma\left(\left(C_{3} \square C_{n}\right)^{2}\right)=\gamma\left(\left(K_{3} \square C_{n}\right)^{2}\right)=\gamma\left(C_{n}\right)=\left\lceil\frac{n}{3}\right\rceil
$$

Theorem 16. $\gamma\left(\left(C_{4} \square C_{n}\right)^{2}\right)=\left\lceil\frac{4 n}{13}\right\rceil, n=4,5,6,7$, and

$$
\gamma\left(\left(C_{4} \square C_{6 k+t}\right)^{2}\right) \leq \begin{cases}2 k & \text { if } t=0, \\ 2 k+1 & \text { if } t=1, \\ 2(k+1) & \text { if } t=2,3,4,5 .\end{cases}
$$

Proof. By Theorem 13 we have $\gamma\left(\left(C_{4} \square C_{n}\right)^{2}\right) \geq\left\lceil\frac{4 n}{13}\right\rceil$. In Figure 6 it is determined by a dominating set of size $\left\lceil\frac{4 n}{13}\right\rceil$ for $\left(C_{4} \square C_{n}\right)^{2}, n=4,5,6,7$, so for these graphs we have $\gamma\left(\left(C_{4} \square C_{n}\right)^{2}\right)=\left\lceil\frac{4 n}{13}\right\rceil$.

In Figure 6, the seventh column of s.d of $C_{4} \square C_{7}$ (from left to right) is similar to the first column of s.d of $C_{4} \square P_{1}, C_{4} \square P_{2}$ and $C_{4} \square C_{n}, n=3,4,5,6,7$. By setting $s . d$ of $k$ graphs $C_{4} \square C_{7}$ and one $s . d$ of $C_{4} \square P_{2}$ or $C_{4} \square C_{3}$ or $C_{4} \square C_{4}$ or $C_{4} \square C_{5}$ consecutively from left to right such that the first column of every s.d of graph locates on the last column of $s . d$ of the previous graph, we can obtain a $s . d$ of $C_{4} \square C_{6 k+t}$ with a dominating set of size $2(k+1)$ for $\left(C_{4} \square C_{6 k+t}\right)^{2}, t=2,3,4,5$. By the same setting for $s . d$ of $k$ graphs $C_{4} \square C_{7}$ we can obtain a s.d of $C_{4} \square C_{6 k+1}$ with a dominating set of size $2 k+1$ for $\left(C_{4} \square C_{6 k+1}\right)^{2}$. Also by the same setting for s.d of $k-1$ graphs $C_{4} \square C_{7}$ and one s.d of $C_{4} \square C_{6} \quad$ we can obtain a s.d of $C_{4} \square C_{6 k}$ with a dominating set of size $2 k$ for $\left(C_{4} \square C_{6 k}\right)^{2}$.

Theorem 17. $\gamma\left(\left(C_{5} \square C_{n}\right)^{2}\right)=\left\lceil\frac{5 n}{13}\right\rceil, n=3,4,6$, and

$$
\gamma\left(\left(C_{5} \square C_{6 k+t}\right)^{2}\right) \leq \begin{cases}3 k & \text { if } t=0, \\ 3 k+1 & \text { if } t=1, \\ 3 k+2 & \text { if } t=2,3,4, \\ 3(k+1) & \text { if } t=5 .\end{cases}
$$

Proof. By Theorem 13 we have $\gamma\left(\left(C_{5} \square C_{n}\right)^{2}\right) \geq\left\lceil\frac{5 n}{13}\right\rceil$. In Figure 7 it is determined by a dominating set for 


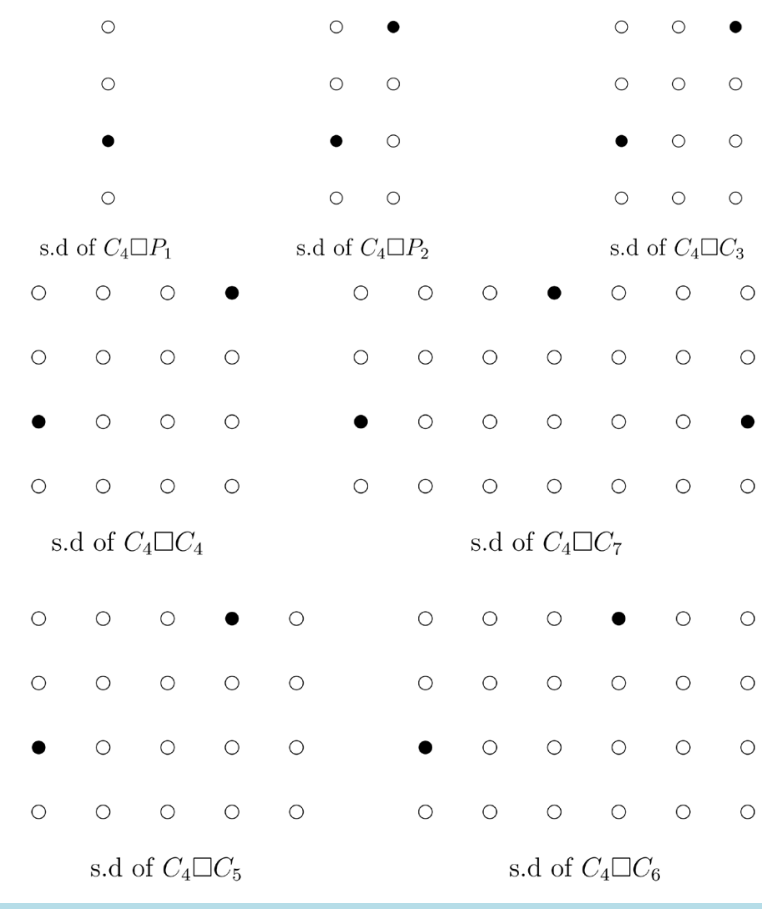

Figure 6. A dominating set for $\left(C_{4} \square C_{n}\right)^{2}, n=1,2, \cdots, 7$.
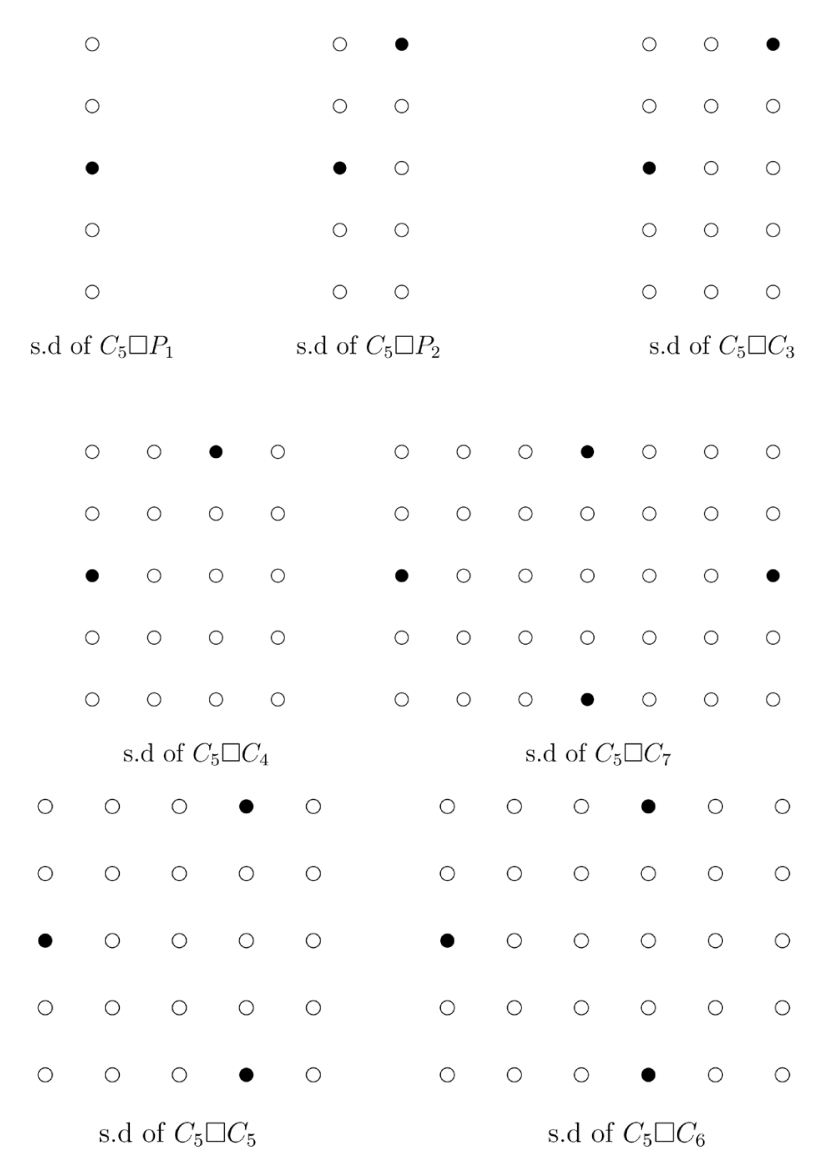

Figure 7. A dominating set for $\left(C_{5} \square C_{n}\right)^{2}, \quad n=1,2, \cdots, 7$. 
$\left(C_{5} \square P_{1}\right)^{2},\left(C_{5} \square P_{2}\right)^{2}$ and $\left(C_{5} \square C_{n}\right)^{2}, n=3,4,5,6,7$.

By Figure 7 we have $\gamma\left(\left(C_{5} \square C_{n}\right)^{2}\right) \leq\left\lceil\frac{5 n}{13}\right\rceil, n=3,4,6$. So for these graphs equality holds.

In Figure 7, the seventh column of $s . d$ of $C_{5} \square C_{7}$ (from left to right) is similar to the first column of s.d of $C_{5} \square P_{1}, C_{5} \square P_{2}$ and $C_{5} \square C_{n}, n=3,4,5,6,7$. By setting s.d of $k$ graphs $C_{5} \square C_{7}$ and one $s . d$ of $C_{5} \square P_{2}$ or $C_{5} \square C_{3}$ or $C_{5} \square C_{4}$ consecutively from left to right such that the first column of every s.d of graph locates on the last column of the previous s.d of graph, we can obtain a s.d of $C_{5} \square C_{6 k+t}$ with a dominating set of size $3 k+2$ for $\left(C_{5} \square C_{6 k+t}\right)^{2}, t=2,3,4$. By the same setting for $s . d$ of $k$ graphs $C_{5} \square C_{7}$ we can obtain a $s . d$ of $C_{5} \square C_{6 k+1}$ with a dominating set of size $3 k+1$ for $\left(C_{5} \square C_{6 k+1}\right)^{2}$ and by the same setting for $s . d$ of $k$ graphs $C_{5} \square C_{7}$ and one s.d of $C_{5} \square C_{5}$ we can obtain a s.d of $C_{5} \square C_{6 k+5}$ with a dominating set of size $3(k+1)$ for $\left(C_{5} \square C_{6 k+5}\right)^{2}$. Also by the same setting for s.d of $k-1$ graphs $C_{5} \square C_{7}$ and one s.d of $C_{5} \square C_{6}$ we can obtain a s.d of $C_{5} \square C_{6 k}$ with a dominating set of size $3 k$ for $\left(C_{5} \square C_{6 k}\right)^{2}$.

\section{References}

[1] West, D.B. (2001) Introduction to Graph Theory. 2nd Edition, Prentice-Hall, Upper Saddle River.

[2] Haynes, T., Hedetniemi, S. and Slater, P.J. (1997) Fundamentals of Domination in Graphs. M. dekker, Inc., New York.

[3] Ore, O. (1962) Theory of Graphs. American Mathematical Society Colloquium Publications, 38 (American Mathematical Society, Providence, RI).

[4] Cockayne, E.J., Ko, C.W. and Shepherd, F.B. (1985) Inequalities Concerning Dominating Sets in Graphs. Technical Report DM-370-IR, Department of Mathematics, University of Victoria.

[5] Walikar, H.B., Acharya, B.D. and Sampathkumar, E. (1979) Recent Developments in the Theory of Domination in Graphs. In MRI Lecture Notes in Math. Mehta Research Institute of Mathematics, Allahabad, Vol. 1.

[6] Vizing, V.G. (1963) The Cartesian Product of Graphs. Vycisl. Sistemy, 9, 30-43. 